\title{
An Energy-Efficient Multipath Routing Protocol for Wireless Sensor Networks
}

\author{
Ye Ming Lu and Vincent W.S. Wong \\ Department of Electrical and Computer Engineering, \\ The University of British Columbia, \\ 2332 Main Mall, Vancouver, Canada, V6T 1Z4 \\ e-mail: $\{y m l u, v i n c e n t w\} @ e c e . u b c . c a$
}

\begin{abstract}
The energy consumption is a key design criterion for the routing protocols in wireless sensor networks. Some of the conventional single path routing schemes may not be optimal to maximize the network lifetime and connectivity. In this paper, we propose a distributed, scalable and localized multipath search protocol to discover multiple node-disjoint paths between the sink and source nodes. We also propose a load balancing algorithm to distribute the traffic over the multiple paths discovered. We compare our proposed scheme with the directed diffusion, directed transmission, and the energy-aware routing protocols. Simulation results show that our proposed scheme has a higher node energy efficiency, lower average delay and control overhead than those protocols.
\end{abstract}

\section{INTRODUCTION}

Wireless sensor networks (WSNs) consist of densely deployed sensor nodes, which have limited computational capabilities, power supply, and communication bandwidth. The potential applications of sensor networks widely span both civil and military domains. Recently, various routing protocols have been proposed for WSNs. Most of them use a single path to transmit data. The optimal path is selected based on the metrics, such as the gradient of information [1], the distance to the destination, or the node residual energy level [2]. Some other routing protocols that use multiple paths [3][4][5] choose the network reliability as their design priority. The data transmission relies mostly on the optimal path. The alternative path is used only when the nodes on the primary route fail. Although the existing single-path approach is flexible, simple and scalable, nodes may deplete their energy supply at a faster rate. This may result in early network partition.

In [6] and [7], a multipath extension of Dynamic Source Routing (DSR) and Ad hoc On-demand Distance Vector (AODV) were proposed to improve the energy efficiency of ad hoc networks by reducing the frequency of route discovery. The directed diffusion [1] is a data-centric routing scheme. The flooding of interest by sinks allows the gradients to be set up within the network. In [5], a multipath routing approach is proposed for the directed diffusion [1] to improve the resilience to node failures. Their work explores the possibility of finding alternate paths connecting the source and sink nodes when node failures occur.

Directed transmission [8] is one of the probabilistic routing techniques, which are derived from the flooding. It uses a retransmission probability function to reduce redundant copies of same event data. The hop distance to the destination and the number of steps that the data packets has traveled are used as parameters. The retransmission control mechanism avoids the intensive usage of the shortest path in a certain level.

The energy-aware routing is proposed in [3]. It uses localized flooding of request messages to find all possible routes between the sources and sinks, as well as the energy costs associated to these paths. In the routing table of the sensor node, every neighbor is associated with a transmission probability, which is computed based on the cost of the path passing through it. The scheme maintains multiple paths but uses only one of them at a time, in order to avoid stressing a particular path and extend the network lifetime.

In [9], the multipath routing is formulated as a linear programming problem with an objective to maximize the time until the first sensor node runs out of energy. The sources are assumed to be transmitting data packets at a constant rate. In [10], the multipath routing is formulated as a constrained optimization problem by using deterministic network calculus.

In this paper, we propose an energy-efficient multipath routing protocol for wireless sensor networks [11]. The contributions of this paper are as follows:

1) We propose a distributed, scalable and localized multipath search algorithm to discover multiple node-disjoint paths between the sink and source nodes.

2) We also propose a load balancing algorithm to distribute the traffic over the multiple paths discovered. The load balancing algorithm allows the sink node to allocate traffic over multiple paths found based on their cost, which depends on the energy levels and the hop distances of nodes along each path.

3) We compare our proposed scheme with the directed diffusion [1], directed transmission [8], and the energyaware routing [3] protocols. Simulation results show that our proposed scheme has a higher node energy efficiency, lower average delay and control overhead than those protocols.

The rest of the paper is organized as follows. Our proposed multipath data routing scheme is described in Section II. The performance evaluation of our scheme as well as the comparisons with other protocols are presented in Section III. Conclusions and future work are given in Section IV. 


\section{Multipath Routing Algorithm}

\section{A. Assumptions and Definitions}

We consider that $M$ identical wireless sensor nodes are distributed randomly in a field. We assume that the network is connected and dense. At any time, a sensor node $m$ is able to acquire the residual energy level $e_{m, \text { residual }}$ of its battery. When a stimulus is detected (or an event occurs), the surrounding nodes first exchange the information and select one of them to be the source node. The source node has the responsibility to aggregate data from the neighboring nodes and to transmit the aggregated data to the sink node. When different events occur in different regions within the coverage area, data from different source nodes are not being aggregated along the path to the sink node.

We define a path, which consists of $K$ nodes, as a group of nodes that relay the data generated from the source node $x$ to the sink node $y$. Since we assume that the network is dense, it is possible to have multiple routes between $x$ and $y$. We assume that the multiple paths used are disjoint. The link cost function is used by the node to select the next hop during the path search phase. Let $N_{a}$ denote the neighbor set of node $a$, node $a$ will choose the next hop by following the criterion:

$$
\arg \min _{b \in N_{a}}\left\{\left(1-e_{b, \text { residual }} / e_{b, \text { init }}\right)^{\left[\beta\left(1-\frac{(\Delta d+1)}{d_{a y}}\right)\right]}\right\}
$$

where $d_{a y}$ is the distance in hops between node $a$ and sink $y ; d_{b y}$ is the distance in hops between node $b$ and sink $y$; $\Delta d$ is the difference between $d_{a y}$ and $d_{b y} ; e_{b, i n i t}$ is the initial energy level of node $b ; e_{b, r e s i d u a l}$ is the residual energy level of node $b$; and $\beta$ is the weight factor and $\beta>1$. Note that $(\Delta d+1) \in\{0,1,2\}$ and $\left(1-e_{b, \text { residual }} / e_{b, \text { init }}\right) \in[0,1]$. The link cost function takes both the node energy level and hop distance into account. Suppose $e_{b, \text { residual }}$ remains constant. In this case, the link cost increases when $(\Delta d+1)$ increases. On the other hand, suppose $(\Delta d+1)$ remains constant. In this case, the link cost increases as $e_{b, r e s i d u a l}$ decreases. The weight factor $\beta$ adjusts the priority. A large $\beta$ gives more weight to the node energy than to the hop distance.

For a path $A$, which consists of $K$ nodes, the path cost $p_{A}$ is the sum of individual link costs $l_{i(i+1)}$ along the path.

$$
p_{A}=l_{12}+l_{23}+\ldots+l_{K(K+1)}
$$

\section{B. Multipath Routing Protocol}

The multipath routing protocol is used to find multiple disjoint paths between a pair of sink and source nodes. It has three phases, the initialization phase, the paths search phase, and the data transmission and paths maintenance phase.

1) Initialization Phase: The HELLO message is first exchanged between the nodes. Fig 1 shows the format of a HELLO message. The field message sequence is a number generated by the message originator. The field message type indicates that it is a HELLO message. The field sender ID contains the node ID of the message originator. The node type field indicates whether the originator is a sink, a source, or

\begin{tabular}{|c|c|c|c|c|c|c|}
\hline $\begin{array}{l}\text { Message } \\
\text { Sequence }\end{array}$ & $\begin{array}{c}\text { Message } \\
\text { Type }\end{array}$ & Sender ID & $\begin{array}{l}\text { Node } \\
\text { Type }\end{array}$ & $\begin{array}{l}\text { Hop } \\
\text { Count }\end{array}$ & $\begin{array}{l}\text { Forward } \\
\text { Node ID }\end{array}$ & $\begin{array}{l}\text { Forward Node } \\
\text { Energy Level }\end{array}$ \\
\hline
\end{tabular}
a regular sensor node. The hop count gives the hop distance

\begin{tabular}{|c|c|c|c|c|c|c|c|}
\hline $\begin{array}{l}\text { Message } \\
\text { Sequence }\end{array}$ & $\begin{array}{c}\text { Message } \\
\text { Type }\end{array}$ & Source ID & Sink ID & Route ID & $\begin{array}{l}\text { Path } \\
\text { Cost }\end{array}$ & $\begin{array}{l}\text { Forward } \\
\text { Node ID }\end{array}$ & $\begin{array}{l}\text { Forward Node } \\
\text { Energy Level }\end{array}$ \\
\hline
\end{tabular}

Fig. 1. The format of a HELLO message.

Fig. 2. The format of a REQUEST message.

of the message that has been passed from its originator. The forward node ID contains the ID of the upstream node, which forwarded the message in the previous hop. The forward node energy level field gives the normalized node energy level of the node that forwarded the message in the previous hop.

When a HELLO message arrives and if it is received for the first time, each node will update its neighboring node table with the forward node ID and forward node energy level. Next, the node verifies if the node type is set to be SINK. In such case, the sender ID is compared with the sink list of the node. A new entry is created in the sink table if necessary, with the hop distance updated only when it is smaller than the value recorded. Finally, the HELLO message from the sink node is re-broadcast with the fields hop count, forward node ID and forward node energy level updated.

At the end of the initialization phase, each node will have the sink table and the neighboring node table updated. Each node then broadcasts a CONNECTIVITY message to its immediate neighbors. This message also includes the field sink numbers to specify the number of sinks that the sender is aware of. The subsequent fields give in order the sink IDs and the hop distance to each of them. The receiving node will update the corresponding entry in its neighboring node table.

2) Paths Search Phase: This phase is initiated when a set of nodes detect the stimulus and the selected source node begins to send the aggregated data to the sink node. The source node unicasts one REQUEST to every neighboring node with a distinct route ID. Fig 2 shows the format of a REQUEST message. The fields source ID and sink ID indicate the node ID of the source and sink, respectively. The route ID is assigned by the source node to distinguish between different routes that lead to the same sink node. The path cost field stores the accumulated path cost, starting from the source node.

Upon reception of REQUEST, a regular node (i.e., an intermediate node) examines its routing table with the values in fields source $I D$ and sink $I D$ and creates a new entry if necessary. If the sink node indicated by sink $I D$ is in the neighboring node table, the routing table is updated and the REQUEST is forwarded to the sink node directly with fields forward ID and forward node energy level updated. Otherwise, the node has to select one of the neighbors to forward the REQUEST. The selection is based on two criteria. First, the neighboring node should not have been selected for another path that connects the same pair of sink and source nodes. Second, the link cost to the selected neighbor has to be the lowest among all the available neighbors. 
The routing table will be updated if a neighbor is selected. The table of neighbors is updated at the same time. In future path search, the node will avoid to select the neighbor that has already been used for the path that connects the same pair of sink and source nodes. Finally, the node will update the fields path cost, forward node ID and forward node energy level before sending the REQUEST to the neighbor selected. If none of the neighbors satisfies the conditions, the REQUEST will simply be dropped.

For the sink, the received REQUEST is processed differently. It first examines the source $I D$ and creates a new entry in its source table if it is not known. It then updates the routing table with the information carried in the message. The sink starts a request timer when it receives the first REQUEST from a source node. The REQUEST messages arrive after the timer expires will simply be dropped. Such measure allows the path exploration to be completed within a reasonable period of time, as REQUEST messages that arrive late will include paths with large delay. When the request timer expires, the sink node begins to allocate traffic to each of the paths discovered. The sink node then sends the ASSIGN messages to the source via each of the selected multipath. This message includes the data transmission rate assigned to each path.

3) Data Transmission and Paths Maintenance Phase: After multiple paths are discovered, the source node begins to transmit data packets with the assigned rates on each path. The DATA message carries the event data and other control fields [11]. At the sink, it updates the path cost in its routing table each time a DATA message arrives. The updated values help the sink node to monitor the conditions of the multiple paths being used. The initial data rate assignments for the paths may not be optimal for the duration of the connection. The sink node has to re-distribute the data rates over paths to optimize the usage of network resources occasionally. The re-distribution is triggered when the original route with the lowest cost has its path cost increased to a pre-determined threshold. The sink node will then adjust the traffic flows and notify the source node with the ASSIGN messages.

In order to detect a path failure, the sink also monitors the inter-arrival delay of data packets on each path. When the delay is above a pre-determined threshold, the sink presumes that the path is broken. If the number of current working paths is equal to or lower than two, the sink will send a RESET message to the source through the optimal path to re-initiate the paths search phase. Otherwise, the sink readjusts the data rate allocation over other functional routes. This mechanism can avoid having the path search phase being invoked frequently.

\section{Load Balancing Algorithm}

We assume that there exists $N$ disjoint paths between a source node $x$ and a sink node $y$. The requested data rate to be arrived at the sink node $y$ via all these multipaths is $R$ bits/sec. Let $r_{j}$ be the data rate allocated to path $j$. For a path $j$, the product of the path cost $p_{j}$ and the data rate allocated $r_{j}$ gives the path cost rate $c_{j}$.
We use the following load balance ratio $\Phi$ (also known as fairness index) to evaluate the level of load balancing over different multipaths:

$$
\Phi(\bar{r})=\frac{\left(\sum_{j=1}^{N} r_{j} p_{j}\right)^{2}}{N \sum_{j=1}^{N}\left(r_{j} p_{j}\right)^{2}}
$$

where the vector $\bar{r}$ denotes the traffic rates allocated to all available routes and $r_{j}$ is the traffic flow allocated to path $j$. The load balance ratio in equation (3) reaches its global maximum of 1 under the condition that the traffic is perfectly balanced. The allocation problem can be formulated as:

$$
\begin{aligned}
& \operatorname{Max} \Phi(\bar{r}) \\
& \text { subject to } \sum_{j=1}^{N} r_{j}=R, \quad \text { where } r_{j} \geq 0
\end{aligned}
$$

To solve the above problem, we first let $k=r_{j} p_{j}$ for all $j$. By substituting $r_{j}=k / p_{j}$ into the constraint, we have

$$
r_{j}=\frac{R}{p_{j}} \sum_{i=1}^{N} p_{i} \quad j=1,2, \cdots N .
$$

\section{PERFormance EVAluation AND COMPARISON}

We implement our multipath routing protocol in the $n s-2$ network simulator and compare it with the directed diffusion [1], the energy-aware routing [3], the directed transmission [8], and the flooding protocols.

\section{A. Simulation Parameters and Performance Metrics}

In all our simulations, we consider a square sensor field of size $L$. Inside the field, $M$ static sensor nodes are deployed randomly. Each node has a fixed radio range of 40 meters. The node density is maintained at a constant level of $50 / 160^{2}$ nodes $/ m^{2}$. The positions of the source and sink nodes are shown in Figure 3. In these configurations, the sinks and sources are located far from each other. The minimum distance between any pair of sink and source is larger than $L / 2$. Such settings facilitate our evaluation of the protocol where the routing path has to traverse a large area in the sensor field. We also assume that the source nodes detect different stimulus. Thus, their event data cannot be aggregated. The data packet size is 64 bytes. The packet data transmission rate is 1 packet per second. We use $\beta=20$ in equation (1).

We adopt the $n s$ - 2 radio energy model and assign each node with the same initial energy level of $10 \mathrm{~J}$ at the beginning of each simulation in order to keep the simulation time within a reasonable time period. We set the initial energy level of the sinks at $40 \mathrm{~J}$ as the sink usually can have its energy supply recharged or replaced in real applications. We further assume that each sensor node carries an omni antenna and the energy consumptions for idle time, transmission and reception are $35 \mathrm{~mW}, 660 \mathrm{~mW}$, and $395 \mathrm{~mW}$ respectively (the same parameters as in [1]). The energy dissipation for data processing in the node is neglected in our simulations. We adopt the IEEE 802.11 MAC layer provided in the $n s-2$ with a bandwidth of $1.6 \mathrm{Mbps}$. Every $500 \mathrm{~ms}$, we obtain the $\log$ of the energy level of each node. This allows us to trace the status of energy consumption of the network. 


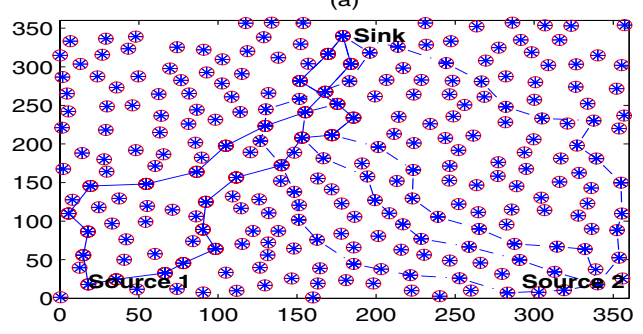

(b)

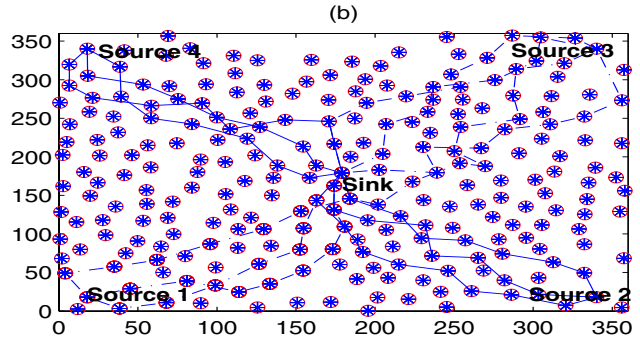

(c)

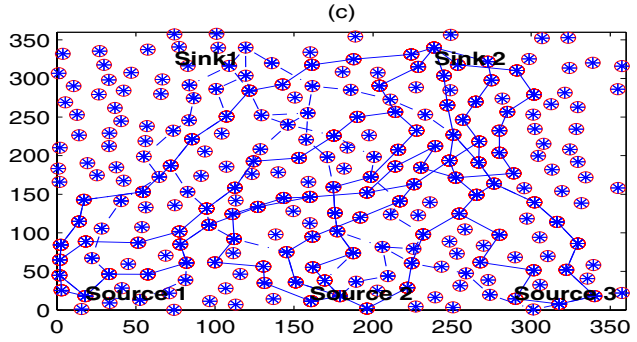

Fig. 3. Configurations of sink and source nodes and examples of paths discovered with 250 nodes deployed (a) Topology setting 1: one sink and two sources (b) Topology setting 2: one sink and four sources (c) Topology setting 3: two sinks and three sources.

The node energy consumption measures the average energy dissipated by the node in order to transmit a data packet from the source to the sink. The same metric is used in [1] to determine the energy efficiency level of WSNs. It is calculated as follows:

$$
\text { node energy consumption }=\frac{\sum_{i=1}^{M}\left(e_{i, \text { init }}-e_{i, r e s}\right)}{M \sum_{j=1}^{S} \operatorname{dataN}_{j}}
$$

where $M$ is the number of nodes, $e_{i, \text { init }}$ and $e_{i, \text { res }}$ are respectively the initial and residual energy levels of node $i$, $S$ is the number of sink nodes and data $N_{j}$ is the number of data packets received by sink $j$. The control message overhead counts the average amount of control messages received and transmitted by each node in bytes. It evaluates the extra workload required to sustain the data routing for various schemes. The average delay measures the average time spent to relay data packets from the source node to the sink node.

\section{B. Results and Discussions}

Fig 4 shows the results for node energy consumption under different topology settings. We can observe that there is a lower node energy consumption of our multipath routing over the other schemes. The flooding is the most costly protocol; by adding a simple mechanism of retransmission probability control on top of the flooding, the directed transmission improves
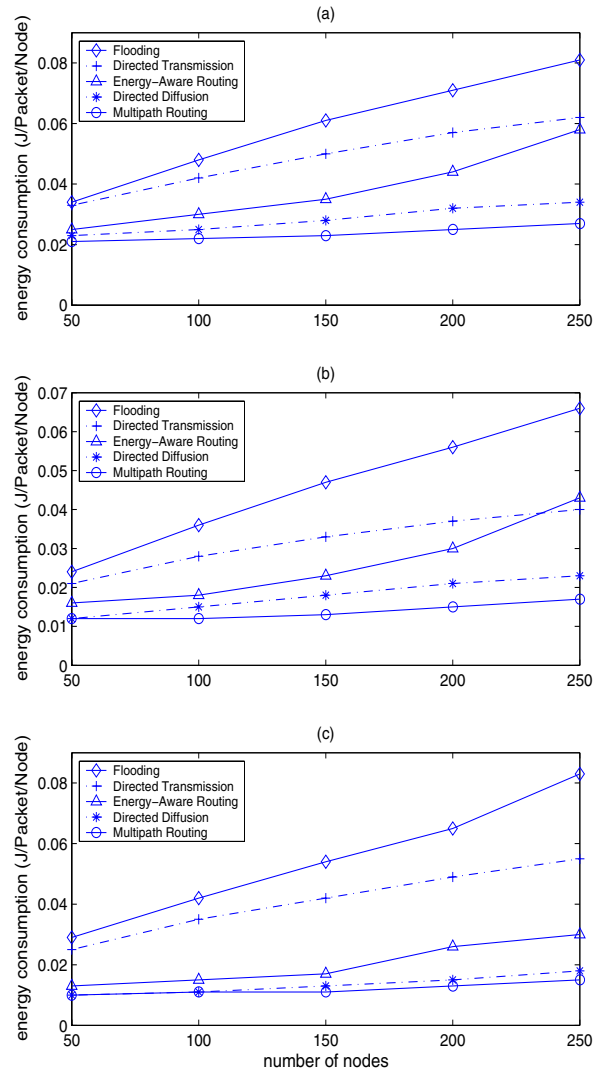

Fig. 4. Average node energy consumption (a) Topology setting 1, (b) Topology setting 2, (c) Topology setting 3.

the energy efficiency. The energy-aware routing obtains further improvement by calculating the retransmission probability as function of the node energy level and the hop distance to the destination. The multipath routing and directed diffusion [1] perform better than other protocols we examined.

In Fig 4(a), the improvement of multipath routing is ranging from $1 \%$ to $34 \%$ when compared with directed diffusion. Such experimental results demonstrate that the energy efficiency of multipath routing is stable and has little impact by the increase of the network size, while the performance of other schemes degrades with larger network size. Fig 4(b) shows a better performance than Fig 4(a) for multipath routing. It is simply due to the difference of topology settings. With 1 sink in the center of the field and 4 sources at four corners in topology 1, the average path length is significantly smaller than that in the topology 2, where one sink and two sources are in opposite edges of the field. As a result, more energy is required to deliver data to the sink in the setting of one sink and two sources.

Fig 5 shows the control message overhead of different protocols. It is obtained by calculating the ratio between the average amount of control message processed by the node and the amount of data packets received by the sinks. The directed diffusion spends much more energy on transmitting and receiving control messages than any other protocols, since it requires periodic interest broadcast and path reinforcement. The multipath routing has a much lower overhead for the 

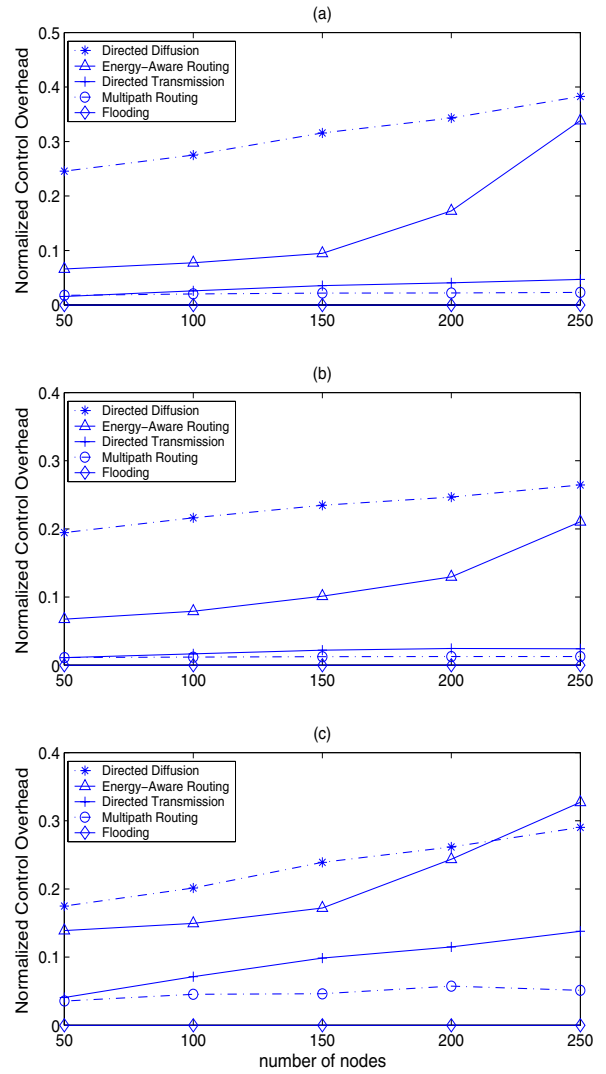

Fig. 5. Ratio of control message overhead vs data traffic received (a) Topology setting 1, (b) Topology setting 2, (c) Topology setting 3.

control message, about $70 \%$ less than the energy-aware routing [3] with the topology setting of 1 sink and 4 sources.

Fig 6 shows the average data transfer delay results. The multipath routing has the shortest delay compared to other schemes. As we expected, data packets are routed through different node-disjoint paths with multipath routing. Hence, the network congestion can be avoided. Further results can be found in [11].

\section{CONCLUSiOnS}

In this paper, we proposed a multipath routing protocol for wireless sensor networks. The distributed multipath routing protocol is capable to search multiple node-disjoint paths. The load balancing algorithm aims to allocate the traffic rate to each path optimally. Simulation results that our proposed scheme has a higher node energy efficiency, lower average delay and control overhead than the directed diffusion, directed transmission, and the energy-aware routing protocols. Further work to improve the algorithm includes the integration of data aggregation and the support of node with limited mobility.

\section{ACKNOWLEDGMENT}

This work is supported by the Natural Sciences and Engineering Research Council of Canada under grant number 261604-03.
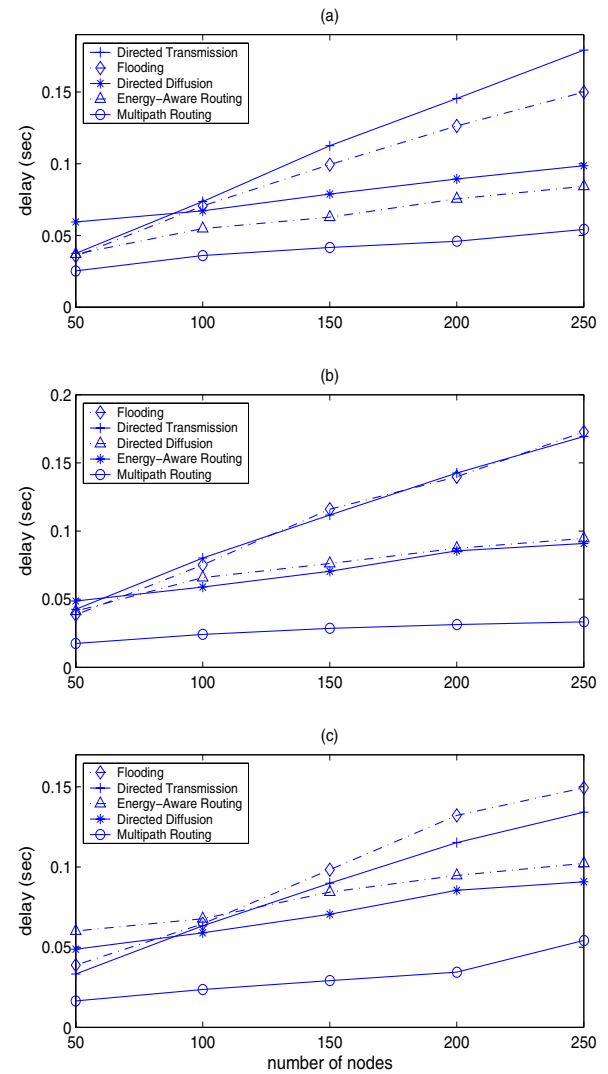

Fig. 6. Average data transfer delay (a) Topology setting 1, (b) Topology setting 2, (c) Topology setting 3 .

\section{REFERENCES}

[1] C. Intanagonwiwat, R. Govindan, and D. Estrin, "Directed diffusion: A scalable and robust communication paradigm for sensor networks," in Proc. of ACM MobiCom'00, Boston, MA, USA, Aug. 2000, pp. 56-67.

[2] Y. Xu, J. Heidemann, and D. Estrin, "Geography-informed energy conservation for ad-hoc routing," in Proc. of ACM/IEEE MobiCom'01, Rome, Italy, July 2001, pp. $70-84$.

[3] R. Shah and J. Rabaey, "Energy aware routing for low energy ad hoc sensor networks," in Proc. of IEEE WCNC'02, Orlando, FL, March 2002, pp. 350-355.

[4] K. Wu and J. Harms, "On-demand multipath routing for ad hoc networks," in Proc. of European Personal and Mobile Communications Conference (EPMCC), Vienna, Austria, Feb. 2001.

[5] D. Ganesan, R. Govindan, S. Shenker, and D. Estrin, "Highly-resilient, energy-efficient multipath routing in wireless sensor networks," in Proc. of ACM MobiHoc'01, Long Beach, CA, USA, Oct. 2001.

[6] A. Nasipuri and S. Das, "On-demand multipath routing for mobile ad hoc networks," in Proc. of International Conference on Computer Communications and Networks (IC3N), Boston, MA, USA, Oct. 1999.

[7] M. Marina and S. Das, "On-demand multipath distance vector routing in ad hoc networks," in Proc. of the Ninth International Conference for Network Protocols (ICNP), Riverside, CA, USA, Nov. 2001.

[8] C. Barrett, S. Eidenbenz, L. Kroc, M. Marathe, and J. Smith, "Parametric probabilistic sensor network routing," in Proc. of ACM WSNA'03, San Diego, CA, Sept. 2003, pp. 122-131.

[9] J. Chang and L. Tassiulas, "Energy conserving routing in wireless adhoc networks," in Proc. of IEEE INFOCOM'OO, Tel-Aviv, Israel, March 2000, pp. 22-31.

[10] S. Mao, S. Panwar, and Y. Hou, "On optimal traffic partitioning for multipath transport," in Proc. of IEEE INFOCOM'05, Miami, FL, March 2005, pp. 2325-2336.

[11] Y. Lu, "Multipath routing algorithm for wireless sensor networks," Master's Thesis, University of British Columbia, Vancouver, Canada, Dec. 2005. 